\title{
Lesões orais associadas ao uso de próteses dentárias: uma revisão da literatura
}

\author{
Oral injuries associated with the use of dental prostheses: a literature review \\ Lesiones orales associadas con el uso de prótesis dentales: revisión de la literatura
}

Recebido: 13/10/2021 | Revisado: 23/10/2021 | Aceito: 25/10/2021 | Publicado: 27/10/2021

\author{
Mayane Souza Santos Silva \\ ORCID: https://orcid.org/0000-0002-1738-2872 \\ Centro Universitário Cesmac, Brasil \\ E-mail: mayane.stop.souza@ hotmail.com \\ João Pedro Lima Xavier \\ ORCID: https://orcid.org/0000-0002-4299-6542 \\ Centro Universitário Cesmac, Brasil \\ E-mail: jplxaju@gmail.com \\ Olivia Maria Guimarães Marroquim \\ ORCID: https://orcid.org/0000-0002-6782-8596 \\ Centro Universitário Cesmac, Brasil \\ E-mail: oliviamarroquim1982@gmail.com \\ João Francisco Tenório Neto \\ ORCID: https://orcid.org/0000-0002-5094-4566 \\ Centro Universitário Cesmac, Brasil \\ E-mail: joaotenorioperio@gmail.com
}

\begin{abstract}
Resumo
Apesar dos avanços na Odontologia, ainda há uma incidência de perdas dentárias, principalmente nos idosos, constituindo um problema de saúde pública. Um dos instrumentos importantes para a reabilitação oral é a prótese, que tem como finalidade reabilitar zonas desdentadas. As consequências do uso inadequado e má higienização de próteses removíveis podem afetar na cavidade oral devido ao acúmulo de microrganismos ou advindas de uma má adaptação, gerando traumatismos sobre os rebordos alveolares. Assim, o objetivo deste estudo é destacar as principais lesões que acometem a cavidade oral decorrentes da má adaptação e pela deficiência de higienização. O estudo trata-se de uma revisão de literatura narrativa, desenvolvida por meio de um levantamento nas bases de dados eletrônicas PubMed, Scholar Google e SciELO. A estratégia de busca se deu por meio dos DeCs "Prótese dentária", "Manifestações bucais" e "Lesões", nos idiomas inglês, espanhol e português. Como critérios de elegibilidade, foram selecionados 20 artigos que possuem temática compatível com os objetivos propostos deste trabalho, excluindo todos os outros. A negligência com à higiene da prótese ou má adaptação pode causar lesões orais, uma vez que é um ambiente propício para proliferação e sobrevivência de microrganismos orais e formação de biofilme. Dentre as patologias mais comuns, pode-se destacar: hiperplasia inflamatória, úlcera traumática, queilite angular e estomatite protética. Conclui-se que a prótese é importante para reabilitar pacientes edêntulos que perderam suas funções essenciais. Contudo, é imprescindível um acompanhamento e uma boa instrução do Cirurgião-dentista sobre higienização oral, além de um ajuste adequado da prótese.
\end{abstract}

Palavras-chave: Prótese dentária; Manifestações bucais; Lesões.

\begin{abstract}
Despite advances in Dentistry, there is still an incidence of tooth loss, especially in the elderly, constituting a public health problem. One of the important instruments for oral rehabilitation is the prosthesis, which aims to rehabilitate edentulous areas. The consequences of inadequate use and poor hygiene of removable dentures can affect the oral cavity due to the accumulation of microorganisms or arising from a poor adaptation, causing trauma to the alveolar ridges. Thus, the aim of this study is to highlight the main injuries that affect the oral cavity resulting from poor adaptation and poor hygiene. The study is a narrative literature review, developed through a survey in the electronic databases PubMed, Scholar Google and SciELO. The search strategy was carried out through the DeCs "Dental prosthesis", "Oral manifestations" and "Lesions", in English, Spanish and Portuguese. As eligibility criteria, 20 articles were selected that have a theme compatible with the proposed objectives of this work, excluding all others. Negligence with the hygiene of the prosthesis or poor adaptation can cause oral injuries, since it is a favorable environment for the proliferation and survival of oral microorganisms and biofilm formation. Among the most common pathologies, we can highlight: inflammatory hyperplasia, traumatic ulcer, angular cheilitis and prosthetic stomatitis. It is concluded that the prosthesis is important to rehabilitate edentulous patients who have lost their essential functions. However, follow-up and good instruction from the dentist on oral hygiene is essential, in addition to an adequate adjustment of the prosthesis.
\end{abstract}

Keywords: Dental phosthesis; Oral manifestations; Injuries. 


\begin{abstract}
Resumen
A pesar de los avances en Odontología, todavía existe una pérdida de piezas dentales, especialmente en los ancianos, lo que constituye un problema de salud pública. Uno de los instrumentos importantes para la rehabilitación bucal es una prótesis, que tiene como zonas rehabilitar áreas edéntulas. Las consecuencias de un uso inadecuado y una mala higiene de las dentaduras postizas removibles pueden afectar a la cavidad bucal por acumulación de microorganismos o derivado de una mala adaptación, provocando traumatismos en las crestas alveolares. Así, el objetivo de este estudio es destacar como las principales lesiones que afectan a la cavidad bucal se originan por una mala adaptación y una mala higiene. El estudio es una revisión de la literatura narrativa, desarrollada a través de una encuesta en las bases de datos electrónicas PubMed, Scholar Google y SciELO. La estrategia de búsqueda se realizó a través de las DeCs "Prótesis dentales", "Manifestaciones orales" y "Lesiones", en los idiomas español y portugués. Como criterio de elegibilidad, se seleccionaron 20 artículos que tienen una temática compatible con los objetivos propuestos de este trabajo, excluyendo todos los demás. La negligencia con la higiene de la prótesis o la mala adaptación pueden provocar lesiones bucales, ya que es un entorno propicio para la proliferación y áreas de microorganismos bucales y formación de biopelículas. Entre las patologías más comunes, podemos destacar: hiperplasia inflamatoria, úlcera traumática, queilitis angular y estomatitis protésica. Se concluye que una prótesis es importante para rehabilitar a pacientes desdentados que han perdido sus funciones esenciales. Sin embargo, es fundamental el seguimiento y una buena instrucción por parte del odontólogo sobre higiene bucal, además de un adecuado ajuste de la prótesis.
\end{abstract}

Palabras clave: Protésis dental; Manifestaciones orales; Lesiones.

\title{
1. Introdução
}

Apesar dos avanços na Odontologia, vale ressaltar que ainda há uma alta incidência de perdas dentárias, principalmente na população idosa, constituindo um sério problema de saúde pública e sendo responsável por consequências físicas e emocionais (Peixoto et al., 2015). A etiologia do edentulismo ocorre principalmente devido a cárie, traumatismos e a doença periodontal, podendo estar correlacionada também às patologias sistêmicas, hábitos do paciente e anomalias como agenesias - unitárias a totais (Trindade et al., 2018).

Conforme Oliveira (2013), a perda total dos dentes é entendida, erroneamente, como um fenômeno natural, sendo um reflexo da falta de prevenção e de orientação, assim como de cuidados com a saúde bucal dos adultos. Esta perda dentária pode causar muitos problemas de saúde - uma vez que a saúde bucal é um indicador fundamental de saúde, bem-estar e qualidade de vida em geral -, como reabsorção óssea maxilomandibular, deficiências nutricionais, problemas psicológicos e de relacionamentos interpessoais (Paraguassu et al., 2019; Escudero et al., 2020).

A baixa eficiência da função mastigatória causa deglutição de pedaços muito grandes de alimentos ou em mudanças significativas na dieta, fazendo com que evite alimentos mais difíceis de serem mastigados (Paraguassu et al., 2019).

Sob esta ótica, Trindade et al. (2018) afirma que um dos instrumentos importantes para a reabilitação oral é a prótese, que tem como finalidade reabilitar zonas desdentadas, podendo ser fixas ou removíveis, parciais ou totais. Atrelado a isso, é imprescindível que antes do planejamento do tratamento haja um bom exame clínico e radiográfico da cavidade oral, onde deve ser avaliado diversos fatores; dentre eles, estão à higiene bucal, condições sistêmicas e funcionalidade da articulação temporomandibular.

As consequências do uso inadequado e má higienização de próteses removíveis podem afetar de forma significativa na cavidade oral, devido ao acúmulo de microrganismos sobre as superfícies ou advindas de uma má adaptação, gerando traumatismos sobre os rebordos alveolares, como as úlceras traumáticas, estomatite protética, queilite angular, hiperplasia inflamatória e candidíase (Trindade et al., 2018; Cunha, 2019).

Diante disso, o objetivo deste estudo é destacar os principais tipos de lesões e patologias que acometem a cavidade oral decorrentes da má adaptação e pela deficiência de higienização por parte do paciente. 


\section{Metodologia}

Trata-se de uma pesquisa descritiva do tipo revisão da literatura narrativa, que busca evidenciar as manifestações orais decorrentes do uso de prótese. A pesquisa foi realizada através do acesso online nas bases de dados Scientific Eletronic Library Online (SciELO), Google Scholar, National Library of Medicine (PubMed MEDLINE), os quais foram de suma importância para entendimento em âmbito global das manifestações orais. Para a busca das obras foram utilizadas as palavras-chaves presentes nos descritores em Ciências da Saúde (DeCS): prótese dentária, manifestações bucais e lesões.

Como critérios de inclusão, foram considerados artigos originais, que abordassem o tema pesquisado, publicados no período de 2013 a 2021, em inglês, português e espanhol. O critério de exclusão foi imposto naqueles trabalhos que não estavam em inglês, português e espanhol que não tinham correlação com o tema. A estratégia de seleção dos artigos seguiu por meio das etapas propostas por Gil (2017): leitura exploratória e reconhecimento dos artigos que interessavam à pesquisa, leitura seletiva, escolha do material que atendeu ao propósito da pesquisa e análise dos textos selecionados. Assim, totalizaram-se 20 artigos científicos para a revisão de literatura narrativa, com os descritores apresentados acima.

\section{Resultados e Discussão}

A reabilitação protética visa devolver ao paciente desdentado as funções fonética, mastigatória e estética, além de proporcionar uma melhora na qualidade de vida (Cunha, 2019). Todavia, o tratamento protético não reduz o aparecimento de novos problemas bucais, os quais, podem ocorrer sobre os elementos biológicos e protéticos envolvidos (Peixoto et al., 2015).

Assim como a dentição permanente necessita de cuidados higiênicos, as próteses também precisam de manutenção e higienização; em casos de negligência quanto à higiene ou pelo fato da mesma não estar adaptada de forma correta na superfície gengival, pode causar lesões orais, uma vez que é um ambiente propício para proliferação e sobrevivência de microrganismos orais e formação de biofilme (Cunha, 2019; Marra et al., 2017).

Dentre as patologias mais comuns em pacientes que fazem o uso de próteses, podem-se destacar: hiperplasia inflamatória, úlcera traumática, queilite angular e estomatite protética.

\subsection{Hiperplasia inflamatória}

A hiperplasia inflamatória está relacionada com a má adaptação das próteses parciais ou totais e/ou por forças oblíquas resultantes de desajustes oclusais, que podem causar traumas constantes e/ou inflamação nos tecidos bucais (Nobre, 2017). Além disso, de acordo com Cunha (2019), é uma das mais comuns de tecido mole, caracterizada por lesão proliferativa e não neoplásica, com um aumento no número de células do tecido conjuntivo, por meio de um agente de irritação com baixa intensidade.

Apresenta-se normalmente assintomático, com aumento volumétrico nodular, localizado às margens das próteses, de consistência flácida, tamanho variado, coloração eritematosa ou clara. Se origina na mucosa gengival, jugal, lingual, lábios e palato, manifestando-se como única ou múltiplas pregas do tecido conjuntivo hiperplásico (Nobre \& Athias, 2017; Oliveira et al., 2021).

\section{2 Úlcera traumática}

A úlcera traumática é uma das lesões mais frequentes da mucosa oral e está correlacionada a um fator irritante local, como próteses mal adaptadas e traumas oclusais (Cunha, 2019). Geralmente, ela se desenvolve após um ou dois dias da instalação da prótese, devido a próteses que não tenham sido adaptadas as condições de rebordo, oclusão não balanceada, próteses mal polidas ou com distorções oclusais (Peixoto et al., 2015). 
A presença de tórus palatino ou sublingual com tamanho exagerado interferem na estabilidade de uma prótese total ou parcial, assim, favorecendo o desenvolvimento das mesmas. Por este motivo, recomenda-se a remoção cirúrgica do tórus, uma vez que pode dificultar a fonação devido as ulcerações frequentes (Peixoto et al., 2015; Oliveira et al., 2021).

Segundo Oliveira et al. (2021), essas lesões são delimitadas, com sintomatologia dolorosa, com área eritematosa recoberta por membrana removível, central, amarela e fibrinopurulenta. Normalmente, ocorre na língua, lábios e mucosa jugal (Neville, 2016).

\subsection{Queilite angular}

A queilite angular (QA) é um processo inflamatório cutâneo, com etiologia multifatorial e que acomete a comissura labial (Oliveira et al., 2021). Sua etiologia é de fatores locais ou sistêmicos predisponentes, são eles: acúmulo de saliva na região, ação irritante de medicamentos, síndrome de Sjogren, síndrome de Down, agentes infecciosos, perca da dimensão vertical em pacientes, com queda do lábio superior sobre o inferior, como ocorre durante o processo de envelhecimento, na ausência de dentes ou próteses mal adaptadas (Cunha, 2019; Oliveira et al., 2021).

Oliveira et al. (2021) também afirma que a lesão pode apresentar-se de forma unilateral ou bilateral, com um espassamento branco acizentado com eritema adjacente. Sua sintomatologia é dolorosa, caracterizada por ardor ou prurido, evolução do eritema, aparecimento de descamação, fissura, úlcera e formação de crosta, as vezes acompanhados por sangramento.

\subsection{Estomatite protética}

A estomatite protética (EP) está localizada na mucosa oral de pacientes que fazem o uso de prótese parcial removíveis ou totais, sendo uma das mais frequentes (Trindade et al., 2018). A EP é considerada uma inflamação da mucosa subjacente à prótese removível, sendo mais associada à região do palato (Moritz \& Coelho, 2018).

Tal lesão pode ser originada por diversos fatores, com causa de origem sistêmica e locais, como o uso contínuo de prótese, porosidade da resina, traumas da mucosa e má higienização (Moritz \& Coelho, 2018). O trauma que é causado por próteses mal adaptadas, falta de higienização da prótese, tabaco, dieta, colonização microbiana - especialmente por Candida albicans - e reação a hipersensibilidade ao material da base está vinculado aos principais fatores causais ou predisponentes (Vitorino et al., 2016).

A EP afeta principalmente a mucosa palatina, na maxila, apresentando-se normalmente assintomática, entretanto, pode causar incômodo ao paciente, por meio do ardor, sensação de queimadura, disgeusia, disfagia, anorexia ou perda de peso (Vitorino et al., 2016).

\section{Considerações Finais}

A prótese é de fundamental importância para a reabilitação oral de pacientes edêntulos, que perderam suas funções essenciais. Contudo, embora seja um aparelho indispensável, é possível observar que há muitas lesões que são derivadas do mesmo, devido ao déficit de higiene oral e má adaptação da prótese.

As manifestações orais podem ser minimizadas por meio de um acompanhamento e uma boa instrução do Cirurgiãodentista sobre higienização oral, além de um ajuste adequado após instalação da prótese, pois dessa forma, irá manter a saúde dos tecidos. É imprescindível enfatizar durante o atendimento que o tratamento continua, pois necessita de visitas periódicas para verificação da higiene bucal e da prótese.

Assim, sugere-se que estudos futuros explorem as lesões orais associadas ao uso de próteses, de modo que a assistência a estes usuários seja garantida. 


\section{Referências}

Cunha, T. O. D. (2019). Patologias recorrentes devido ao uso de prótese total. [Dissertação para obtenção do título de Bacharel em Odontologia]. Centro Universitário do Planalto Central Apparecido dos Santos.

Bitencourt, F. V., Corrêa, H. W., \& Toassi, R. F. C. (2019). Experiências de perda dentária em usuários adultos e idosos da Atenção Primária à Saúde. Ciênc. saúde colet., 24(1).

da Silva, R. S., da Silva, R. S., Moreira, C. L., Marques, J. G., Nascimento, M. A., Baruta, A. C. G., \& Moris, D. V. (2018). Prevalência de espécies de Candida spp. isoladas da cavidade oral e próteses dentárias removíveis de pacientes atendidos em clínica Odontológica. In Colloquium Vitae, 10 (1), 52-57.

de Melo, I. A., \& Guerra, R. C. (2014). Candidíase oral: um enfoque sobre a estomatite por prótese. Salusvita, 33(3), 389-414.

Eler, K. A. S., Kaiser, T. F., \& Batista, C. C. S. (2017). Candidíase em pacientes portadores de prótese total. [Dissertação para obtenção do título de Bacharel em Cirurgião-dentista]. Centro Universitário São Lucas.

Escudero, E., Rentería, M. V. M., Claure, M. L. C., Justiniano, L. A., \& Mobarec, E. Y. V. (2020). Prevalencia del edentulismo parcial y total, su impacto em la calidad de vida de la población de 15 a 85 años de sucre, 2019. Revista Ciência, Tecnologia e Inovação, 18(21), 161-190.

Fernandes, A. B. F., Garcia, A. V., Gugelmin, B. P., Kintopp, C. A., Pizzato, E., Machado, M. A. N., \& Gabardo, M. C. L. (2020). Impacto del uso de prótesis totais en la calidad de vida de los ancianos institucionalizados. Rev Cubana Estomatol, 57(1).

Freire, J. C. P., Nóbrega, M. T. C., Freire, S. C. P., \& Dias-Ribeiro, E. (2017). Candidíase oral em usuários de próteses dentárias removíveis: fatores associados. Arch Health Investe, 6(4), 159-161.

Gil, R. L. (2017). Como elaborar projetos de pesquisa, $6^{\text {a }}$ edição. São Paulo: Atlas.

Gomes Filho, V. V., Moreira, R. F., Silva Junior, M. F., Gondinho, B. V. C., Cavalcante, D. F. B, Bulgareli, J. V., Guerra, L. M., Frias, A. C., Meneghim, M. C., \& Pereira, A. C. (2020). Fatores associados à necessidade de prótese total em um ou ambos os arcos na população idosa. Brazilian Oral Research, 34(1).

Machado, C. S., Silva, K. T., Nadal, L., Fosqueira, E. C., \& Boleta-Ceranto, D. C. F. (2015). Manifestações orais e implicações odontológicas em portadores de Diabetes Mellitus na região de Cascavel - PR. Revista UNINGÁ Review, 23(2), 05-10. Original Research Community Dentistry, 23(2), 05-10.

Marra, J., Perez, L. E. C., Henriques, T. E., Pinheiro, M. T., \& Castro, F. L. A. (2017). Avaliação da correlação entre o grau de instruções e qualidade de higiene de usuários de próteses totais com a presença de estomatite protética. Rev Odontol Bras Central, 26(76), 15-20.

Moritz, P. B., \& Coelho, L. S. B. (2018). Manifestações orais associadas ao uso de próteses removíveis mal adaptadas. [Dissertação para obtenção do Grau de Mestre em Medicina]. Instituto Universitário de Ciências da Saúde.

Neville, B. W.; Damm, D. D.; Allen, C. M., \& Bouquot, J. E (2016). Patologia Oral e Maxilofacial. Elsevier.

Nobre, I. B. B., \& Athias, R. B. (2017). Lesões bucais causadas pelo uso de próteses dentárias removíveis. [Dissertação para obtenção do título de Bacharel em Cirurgião-dentista]. Centro Universitário São Lucas.

Oliveira, G. S., MELO, S. B. S., \& Castro, D. T. (2021). Lesões da mucosa oral associadas ao uso de próteses removíveis. [Dissertação para obtenção do Grau de Graduada em Odontologia]. Universidade de Uberaba.

Oliveira, F. T. S. (2013). O impacto do edentulismo na qualidade de vida de idosos. [Dissertação para obtenção do Grau de Especialista em Atenção Básica em Saúde da Família]. Universidade Federal de Minas Gerais.

Paraguassu, E. C., Figueira, K. S., Lacerda, J. P., Guimarães Neto, U. G., \& Gomes, C. E. V. (2019). Qualidade de vida e satisfação em usuários de prótese total no estado do Amapá, Brasil. Revista Eletrônica Acervo Saúde, 27(1).

Pardini, G., Leão, A. T. T., \& Penoni, D. C. (2019). Uma investigação sobre perda dentária em pacientes geriatras: um estudo transversal. Revista Naval de Odontologia, 46(1), 14-20.

Peixoto, A. P. T., Peixoto, G. C., \& Alessandretti, R. (2015). Relação entre o uso de prótese removível e úlcera traumática - revisão de literatura. J Oral Investe, 4(1), 26-32.

Trindade, M. G. F., Oliveira, M. C., Prado, J. P., \& Santana, L. L. P. (2018) Lesões associadas à má adaptação e má higienização da prótese total. Id on Line Ver. Mult. Psic., 12(42), 956-968.

Vitorino, L. A. M., Carvalho, J., \& Felix, S. (2016). Prevalência de lesões orais associadas ao hábito de dormir com prótese removível. [Dissertação para obtenção do Grau de Mestre em Medicina Dentária]. Instituto Superior de Ciências da Saúde Egas Moniz. 